\title{
West Nile Virus: Epidemiological and Surveillance Approaches
}

\author{
Hammad Akram* \\ Graduate, Hamdard College of Medicine \& Dentistry, Karachi, Pakistan
}

\section{*Correspondence to Hammad Akram, MD, MPH; Graduate, Hamdard College of Medicine \& Dentistry, Karachi, Pakistan. \\ Tel: 01197440399008 ; Email: hammadakram77@yahoo. com}

Received September 19, 2017 Accepted September 26, 2017 Published online September 31 2017

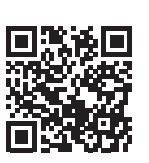

Please cite this article as follows: Akram H. West Nile virus: epidemiological and surveillance approaches. Int J Basic Sci Med. 2017;2(3):111112. doi:10.15171/ ijbms.2017.21.

\section{Dear Editor,}

The West Nile virus (WNV) is one of the notifiable or reportable vector-borne conditions. Human infections are usually asymptomatic; however, it can result in mild to severe illness especially among older population and individuals with immune deficiency. ${ }^{1}$ Since there is no direct treatment or vaccination against human WNV infections, efficient public health and epidemiological response could be critical in preventing and/or controlling the disease.

The West Nile virus outbreak of 2012 is considered one of the most significant events in the United States. The WNV is a flavivirus that can cause morbidity and often mortality due to the neurological disease. ${ }^{1}$ Most of the infected individuals do not present any symptoms; however, when they do, they can also have milder illness known as WNV fever or severe form of the disease that can lead to meningitis or encephalitis also known as the neuroinvasive disease. ${ }^{1}$ The chances of getting severe or fatal disease are higher among individuals who are older than 50 years of age and/or those with weak immunity due to any underlying conditions. The virus is transmitted through mosquito bite making WNV a vector-borne disease. The disease was reported in the West Nile district of Uganda in 1937 and identified in the United States in 1999.

The WNV is one of the reportable conditions by Center for Disease Control and Prevention. ${ }^{2}$ The role of a public health agency to carry out an epidemiological response to any notifiable or reportable disease. This includes performing disease surveillance, case tracking, laboratory data collection, patient information review, establishing the case definition, interviewing the identified cases, maintaining the database. During WNV case investigation, it is important to collect information pertaining to the demographics, history of travel, outdoor activity, and recent mosquito bite among identified patients. After extracting the information from the medical records and client interviews, a line list could be maintained to observe the data by key demographic indicators and exposure indicators which would be very useful in identifying the high-risk regions for disease within the county. Case mapping or identification and marking of confirmed cases on a map are essential in targeting control measures and health promotion activities by geographical regions. The health promotion and preventive activities include educating the community to use approved mosquito repellents every time they go outside, draining of stagnant water and wearing appropriate clothing such as long sleeves and pants when outside and during dusk and dawn times when mosquitoes are more active. ${ }^{3}$

Effective control and response to WNV and any other reportable disease is to have efficient surveillance systems in place. In order to assess and improve the surveillance systems, qualitative, quantitative and other epidemiological approaches can be used, the example of which is shown in Figure 1. This surveillance assessment and capability can be assessed by formulating multiple phases or projects. In current example, a 3 phase process is discussed. In phase 1 , qualitative approaches could be utilized to understand the established surveillance capacity within

Copyright $(2017$ The Author(s); Published by Zabol University of Medical Sciences. This is an open-access article distributed under the terms of the Creative Commons Attribution License (http://creativecommons.org/licenses/by/4.0), which permits unrestricted use, distribution, and reproduction in any medium, provided the original work is properly cited. 


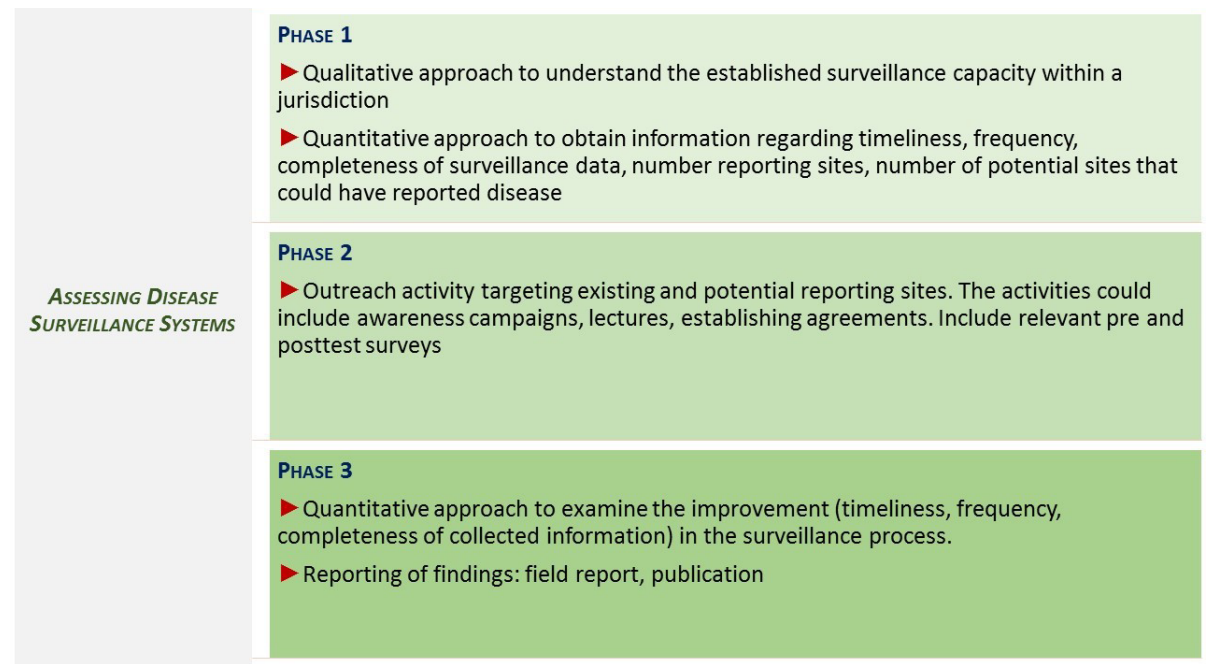

Figure 1. Assessing Disease Surveillance Systems.

a jurisdiction. During same phase, quantitative methods could be implemented to collect baseline information to examine timeliness, frequency, completeness of existing surveillance process and capacity. This can be quantified based on the number of disease reporting sites, number of potential sites that could have reported diseases, and total number of possible reporting sites in a given jurisdiction. In phase 2, outreach activities targeting existing and potential reporting sites could be implemented. The activities could include awareness campaigns, lectures, establishing agreements and so on. Relevant pre- and post-test surveys to assess knowledge attitude and behavioral aspects of potential sites could be essential at this point as a part of project measurement process. In phase 3, a quantitative approach could be used to examine the improvement (timeliness, frequency, completeness of collected information) in the surveillance process. Final phase can include the dissemination of findings through a field report and scientific publications, so the methods and approaches could be used in other jurisdiction to enhance their surveillance systems. Along with the assessment of surveillance systems, other approaches to enhance public health response by involving professionals with diverse skill-sets could play an essential role in early detection and control of diseases and implementation of health promotion initiatives. ${ }^{4}$

\section{Ethical Approval}

Not applicable.

\section{Competing Interests}

Authors declare that they have no potential conflict of interests.

\section{References}

1. World Health Organization, Media Center, West Nile Virus. http://www.who.int/mediacentre/factsheets/fs354/ en/. Accessed August 17, 2017. Published 2011.

2. Center for Disease Control and Prevention, MMWR, West Nile Virus and Other Nationally Notifiable Arboviral Diseases - United States. https://www.cdc.gov/mmwr/ volumes/66/wr/mm6602a3.htm. Accessed August 8, 2017. Published 2015.

3. Center for Disease Control and Prevention, West Nile Virus, Prevention. https://www.cdc.gov/westnile/prevention/ index.html. Accessed August 23, 2017. Published 2017.

4. Akram H. Enhancing public health capacity by using epidemiological teams in a public health setting. Cureus. 2017;9(6):e1381. doi:10.7759/cureus.1381. 